\title{
Fuß und Bein ihre Erkrankungen und deren Behandlung
}

\author{
Ein Lehrbuch \\ von \\ Prof. Dr. med. Georg Hohmann \\ Direktor der Orthopädischen Universitäts-Klinik \\ Frankfurt a. M.
}

Dritte Auflage

Mit 405 Abbildungen

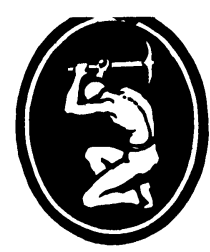

Springer-Verlag Berlin Heidelberg GmbH 1939 
ISBN 978-3-662-40489-8

ISBN 978-3-662-40966-4 (eBook)

DOI 10.1007/978-3-662-40966-4

Alle Rechte, insbesondere das der Ubersetzung in fremde Sprachen, vorbehalten.

Copyright 1934 and 1939 by Springer-Verlag Berlin Heidelberg

Ursprünglich erschienen bei J. F. Bergmann in München 1939

Softcover reprint of the hardcover 3rd edition 1939 
Seinem lieben und verehrten Lehrer

Geheimrat

\section{Professor Dr. Fritz Lange}

zum 75. Geburtstage

gewidmet 


\section{Vorwort zur dritten Auflage.}

In den letzten 5 Jahren ist wieder viele fruchtbare Arbeit an dem Fuß. problem geschehen. Das Interesse der Wissenschaft ist größer geworden, aber auch die Menschen hören mehr auf das, was die Ärzte über Fußschäden, deren Ursachen, Verhütung und Behandlung sagen. Die Schuhmacher wollen ihren Berufsstand heben. Darum hat der Reichsinnungsmeister des deutschen Schuhmacherhandwerks A. Hess die Berufsbezeichnung: orthopädischer Schuhmacher an eine wissenschaftliche und praktische Fortbildung und Prüfung gebunden, die von der Frankfurter orthopädischen Klinik zum ersten Male durchgeführt wurde. Auch die Schuhindustrie ist zur Mitarbeit mit den orthopädischen Ärzten bereit. Der Schuh als Förderungs- oder Hemmungsmittel der Gesundheit und Leistungsfähigkeit des Fußes steht im Mittelpunkt tätigen Interesses. Eine Prüf- und Forschungsstelle für Schuh- und Leistenbau ist unter amtlicher Mitwirkung ins Leben getreten. Neue Schuhformen und -typen werden erprobt. Der alte Petrus Camper würde sich freuen über das nun endlich erwachte Interesse für Fuß und Schuh.

Dieses Buch will nicht einer geringen Sache dienen. Arbeits- und Wehrfähigkeit eines Volkes hängen zu einem guten Teil auch von seiner Fußgesundheit ab. Vieles in dieser Ausgabe ist neu bearbeitet, vertieft und gemäß neuer Erkenntnis erweitert und geändert. Das Bildmaterial ist sehr vermehrt. Das Wesentliche unserer heutigen Anschauung über die Fußfragen ist die bewußte und eifrige Bemühung, den Kampf gegen die Fußschwäche so früh wie möglich zu beginnen, das heißt ihn als einen Teil der allgemeinen körperlichen Erziehung aufzufassen und durch eine richtige Pflege des Fußes von Anfang an die Entstehung von Fußschäden zu verhindern, im Sinne der Gesundheitsführung des Staates.

Trotz des Wunsches von Kritikern habe ich den Klumpfuß oder die paralytischen Deformitäten nicht mit aufgenommen, weil diese Erscheinungen eine umfassende Darstellung verdienen, die den Raum dieses Buches überschreitet.

Auch diese 3. Auflage widme ich meinem lieben und verehrten Lehrer FRITz LANGE, der im Juni seinen 75. Geburtstag begeht. Er hat seinerzeit in mir die Lust, mich mit dem Fuße zu beschäftigen, geweckt und nimmt an dem Fortgang dieser Arbeiten weiter Anteil. Eine Frucht seiner Anregungen ist auch das Buch: Fuß und Bein.

Frankfurt a. M., Ostern 1939. 


\section{Inhaltsverzeichnis.}

1. Bau und Funktion des Fußes und Beines . . . . . . . . . . . . . . . . 1

a) Der Bau . . . . . . . . . . . . . . . . . . . . . . . . . . . . . . . 1

b) Das Skelet . . . . . . . . . . . . . . . . . . . . . . . . . . . . 3

c) Die Bänder . . . . . . . . . . . . . . . . . . . . . . . . . . . . . . 6

d) Die Muskeln . . . . . . . . . . . . . . . . . . . . . . . . . . . . . 7

e) Die Funktion . . . . . . . . . . . . . . . . . . . . . . . . . . . . . . 18

2. Die Untersuchungsmethoden . . . . . . . . . . . . . . . . . . . . . . . 32

3. Die Pflege des Fußes . . . . . . . . . . . . . . . . . . . . . . . . . . . 36

4. Der Knickfuß und Knickplattfuß . . . . . . . . . . . . . . . . . . . . . 39

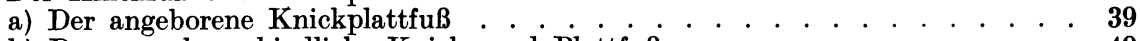

b) Der erworbene kindliche Knick- und Plattfuß . . . . . . . . . . . . . . . . 49

c) Der rachitische Knickplattfuß . . . . . . . . . . . . . . . . . . . . . 54

d) Beschwerden beim kindlichen Knickfuß . . . . . . . . . . . . . . . . . . . . 56

e) Das Einwärtsgehen der Kinder . . . . . . . . . . . . . . . . . . . . . 57

f) Der Fuß im Adoleszentenalter . . . . . . . . . . . . . . . . . . . . . 58

g) Der Fuß im Erwachsenenalter . . . . . . . . . . . . . . . . . . . . . 60

h) Der Senkfuß des Erwachsenen . . . . . . . . . . . . . . . . . . . . . 63

i) Der contracte Plattfuß . . . . . . . . . . . . . . . . . . . . . . . . . . 63

k) Der schwer deformierte Plattfuß . . . . . . . . . . . . . . . . . . . . . 64

l) Die Kontraktur des vorderen unteren Sprunggelenks . . . . . . . . . . . . . . . . . 69

m) Der Werdegang des Knickplattfußes . . . . . . . . . . . . . . . . . . . 75

n) Die Muskeln im Werdegang des Knickplattfußes . . . . . . . . . . . . . . . 79

o) Die Fußspur ... . . . . . . . . . . . . . . . . . . . . . . . . . . 83

p) Die Meßverfahren . . . . . . . . . . . . . . . . . . . . . . . . . . . . . . .85

q) Die Behandlung des Knickplattfußes . . . . . . . . . . . . . . . . . . . . . . . . 88

๔) Die Vorbeugung . . . . . . . . . . . . . . . . . . . . . . . . . . 88

B) Konservative Maßnahmen . . . . . . . . . . . . . . . . . . . . . . 90

y) Übung und Massage . . . . . . . . . . . . . . . . . . . . . . . . 91

1. Übungen für die kurzen Fußmuskeln . . . . . . . . . . . . . . . . . . . . . 93

2. Isolierte Übungen für die supinatorischen Muskeln

3. Gang-, Stand-, Springübungen . . . . . . . . . . . . . . . . . . 96

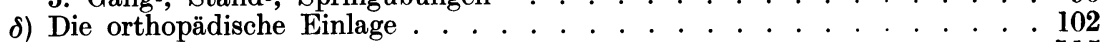

ع) Der Schuh . . . . . . . . . . . . . . . . . . . . . . . . . . . . . 115

६) Verbände. Der Heftpflasterverband . . . . . . . . . . . . . . . . . . . . . . . . . 128

7) Operative Behandlung . . . . . . . . . . . . . . . . . . . . . . . . 131

5. Hallux valgus und Spreizfuß . . . . . . . . . . . . . . . . . . . . . . . 146

a) Wesen und Ursachen . . . . . . . . . . . . . . . . . . . . . . . . 146

b) Behandlung . . . . . . . . . . . . . . . . . . . . . . . . . . . . . . . . . . . .9157

6. Metatarsalgie. (Mortonsche Neuralgie, Fußgeschwulst, Deutschiandersche Erkrankung des Mittelfußes) . . . . . . . . . . . . . . . . . . . . . . . 183

7. Ballenfuß, Hohlfuß, Hackenfuß . . . . . . . . . . . . . . . . . . . . . . 193

8. Die Malacie der Metatarsalköpfchen (A. KöHLER II) . . . . . . . . . . . . . . . . . 203

9. Arthrosis und Arthritis deformans der Fußgelenke. . . . . . . . . . . . . . 211

10. Arthrosis und Arthritis deformans im Kniegelenk . . . . . . . . . . . . . . 218

a) Die Ursachen ... . . . . . . . . . . . . . . . . . . . . . . . . . . . . . . .219

b) Die Krankheitserscheinungen . . . . . . . . . . . . . . . . . . . . . . . . . . . 225

11. Arthrosis und Arthritis deformans des Hüftgelenks . . . . . . . . . . . . . 227

12. Die Behandlung der Arthrosen und Arthritiden . . . . . . . . . . . . . . . 233

a) Allgemeine Maßnahmen . . . . . . . . . . . . . . . . . . . . . . . . 233

b) Lokale Maßnahmen . . . . . . . . . . . . . . . . . . . . . . . . . . 234

c) Das Hüftgelenk . . . . . . . . . . . . . . . . . . . . . . . . . . . . . . . . . . . . . . . . 237

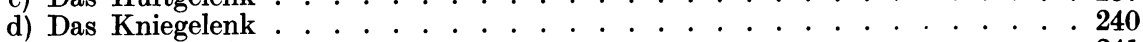

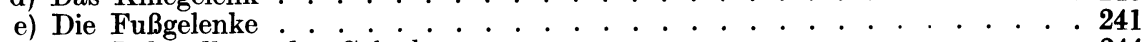

f) Die Behandlung des Gelenkergusses . . . . . . . . . . . . . . . . . . . 244 
13. Hallux rigidus oder flexus . . . . . . . . . . . . . . . . . . . . . . . . 245

14. Hallux malleus . . . . . . . . . . . . . . . . . . . . . . . . . . . . . 260

15. Beugekontraktur der Großzehe . . . . . . . . . . . . . . . . . . . . . . 261

16. Gichtische Gelenkerkrankungen . . . . . . . . . . . . . . . . . . . . . . . . 262

17. Hammerzehen, Krallenzehen, Klauenzehen, Varuszehen . . . . . . . . . . . 263

a) Die angeborenen Formen . . . . . . . . . . . . . . . . . . . . . . . . 263

b) Die erworbenen Formen . . . . . . . . . . . . . . . . . . . . . . . . . . . . . . . 264

c) Der Hammerzehen-Plattfuß . . . . . . . . . . . . . . . . . . . . . . 274

d) Verkrümmung der 5. Zehe . . . . . . . . . . . . . . . . . . . . . . . . . . . . . 277

18. Der untere Fersensporn . . . . . . . . . . . . . . . . . . . . . . . . . . 279

19. Andere krankhafte Zustände an der Ferse . . . . . . . . . . . . . . . . . 286

a) Achillotendinitis . . . . . . . . . . . . . . . . . . . . . . . . . . . . 287

b) Verknöcherungen . . . . . . . . . . . . . . . . . . . . . . . . . . . . . . . . . . . . .289

c) Der hintere Fersensporn . . . . . . . . . . . . . . . . . . . . . . . . . . . . . . . . . . . . 290

d) Rheumatische und unspezifische Entzündungen mit ossifizierender Periostitis 291

e) Spezifische Entzündungen . . . . . . . . . . . . . . . . . . . . . 297

f) Behandlung . . . . . . . . . . . . . . . . . . . . . . . . . . . . . . 297

g) Haquund Exostose mit Bursitis Achillea . . . . . . . . . . . . . . . . . . . . . 299

h) Decubitusnarben am Fersenbein . . . . . . . . . . . . . . . . . . . . 302

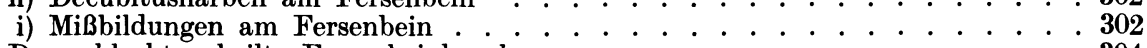

20. Der schlecht geheilte Fersenbeinbruch . . . . . . . . . . . . . . . . . . . . 304

21. Wachstumsstörungen an Fuß und Bein . . . . . . . . . . . . . . . . . . . 305

a) Apophysitis calcanei . . . . . . . . . . . . . . . . . . . . . . . . . . . . . . . . 306

b) A. KöHLERsche Erkrankung des Kahnbeins . . . . . . . . . . . . . . . . . 310

c) Wachstumsstörungen der Epiphysen am Sprunggelenk . . . . . . . . . . . . . . 314

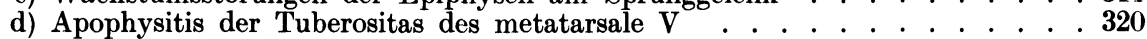

e) Epiphysitis der proximalen Metatarsalepiphysen des Fußes . . . . . . . . . . . 320

f) SchlatTer-Osgoodsche Erkrankung der Tuberositas tibiae . . . . . . . . . . 321

g) Osteochondritis coxae deformans juvenilis . . . . . . . . . . . . . . . . . . 323

22. Akzessorische Knochenstücke am Fuß . . . . . . . . . . . . . . . . . . . . . . . . . .

23. Processus trochlearis calcanei . . . . . . . . . . . . . . . . . . . . . . . . . . 333

24. Sehnenscheidenentzündung am $\mathrm{Fu} \beta$ und Unterschenkel . . . . . . . . . . . . . . . 335

25. Angeborene Fehlbildungen des Fußes . . . . . . . . . . . . . . . . . . . 337

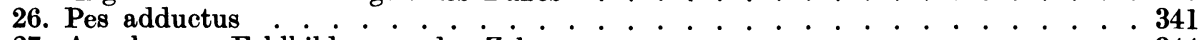

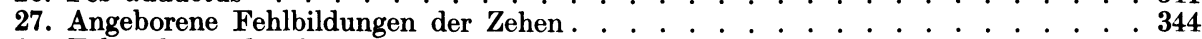

28. Erkrankung der Sesambeine des 1. Mittelfußzehengelenks. . . . . . . . . . . . . . . . . . . 349

29. Der dorsale Knochenhöcker am 1. Keilbein-Mittelfußknochengelenk . . . . . . . . . 352

30. Die Muskelhärten . . . . . . . . . . . . . . . . . . . . . . . . . . . . 355

31. Distorsion des Sprunggelenks

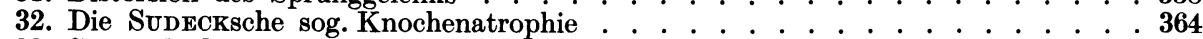

33. Sportschäden an Fuß und Bein . . . . . . . . . . . . . . . . . . . . . . 370

a) Úberanstrengungsschäden . . . . . . . . . . . . . . . . . . . . . . . . . . . 537

b) Sportverletzungen . . . . . . .

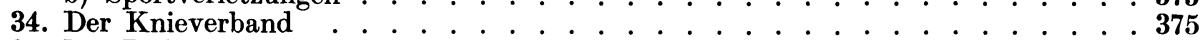

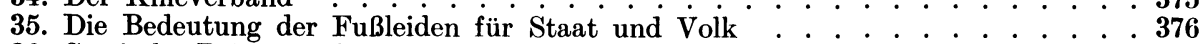

36. Statische Beinveränderungen durch Beinverkürzung . . . . . . . . . . . . . . . . . . . 378

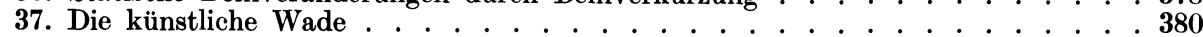

38. Das intermittierende Hinken oder die Gangstockung. . . . . . . . . . . . . . . . . . 381

39. Nervenstörungen am Fuße . . . . . . . . . . . . . . . . . . . . . . . . . . . . . 386

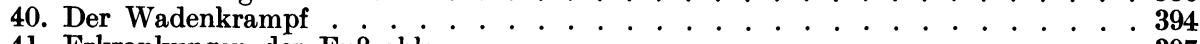

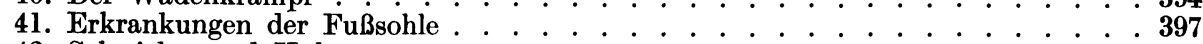

42. Schwielen und Hühneraugen . . . . . . . . . . . . . . . . . . . . . . . . . . . . . . . . . . . 399

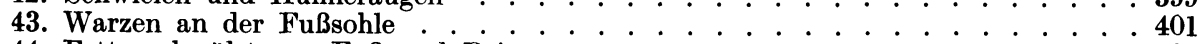

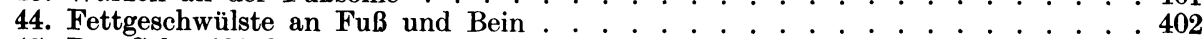

45. Der Schweißfuß . . . . . . . . . . . . . . . . . . . . . . . . . . . . . . . . . . 403

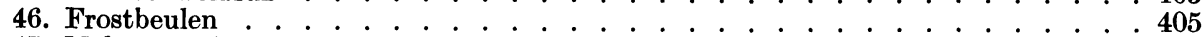

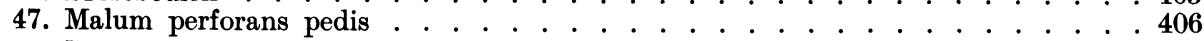

48. Der eingewachsene Nagel . . . . . . . . . . . . . . . . . . . . . . . . . . . . . . . . . 409

49. Krampfadern, Beingeschwür und Venenentzündung . . . . . . . . . . . . . . . . . 415

Die Venenentzündung . . . . . . . . . . . . . . . . . . . . . . . . . 428

50. Die dicken Beine . . . . . . . . . . . . . . . . . . . . . . . . . . . . 432

51. Hämangiome der Wadenmuskulatur . . . . . . . . . . . . . . . . . . . . . . . . . . . . . 4436

52. Der Zinkleimverband . . . . . . . . . . . . . . . . . . . . . . . . . . . . . . 438

Schrifttumsverzeichnis . . . . . . . . . . . . . . . . . . . . . . . . . . . 443

Sachverzeichnis . . . . . . . . . . . . . . . . . . . . . . . . . . . 461 